\title{
Programming cellular function
}

\author{
Christopher A Voigt \& Jay D Keasling
}

\begin{abstract}
The process of cellular engineering is rapidly accelerating owing to advances in technologies to manipulate DNA and other biomolecules, giving rise to the field of synthetic biology. A meeting was held in August 2005 to present progress in the field and to discuss topics in ethics, safety and security.
\end{abstract}

An aim of synthetic biology is to program new cellular functions using well-characterized genetic components. This requires the construction of synthetic sensors that receive environmental information, new circuits to integrate and interpret the signals and mechanisms to link the circuit output to control cellular processes. The time required to design, construct and test a synthetic organism is declining owing to several central technologies. Large-scale genome sequencing projects have provided a toolbox of microbial genetic components that can be swapped between organisms and combined. In addition, plummeting DNA synthesis costs are rendering the slow processes of cloning and molecular biology obsolete. Finally, there is a new effort to standardize genetic parts to improve the predictability of designs and facilitate the exchange of materials between research groups. These technologies will drive new applications and will require rethinking the ethical, safety and security underpinnings of genetic engineering.

A two-day Life Engineering Symposium was held on August 19-20, 2005 to address recent advances in synthetic biology. The symposium was held at the University of California, San Francisco's (UCSF) Mission Bay Campus and was cosponsored by the Keck/National Academies Futures Initiative, the California

Christopher A. Voigt is in the Department of Pharmaceutical Chemistry and California Institute for Quantitative Biomedical Research (QB3), University of California, San Francisco, San Francisco, California 94143-2540, USA.

Jay D. Keasling is in the Department of Chemical Engineering and the Berkeley Center for Synthetic Biology at the University of California, Berkeley, Berkeley, California 94720-3224, USA. e-mail: cavoigt@picasso.ucsf.edu or keasling@berkeley.edu

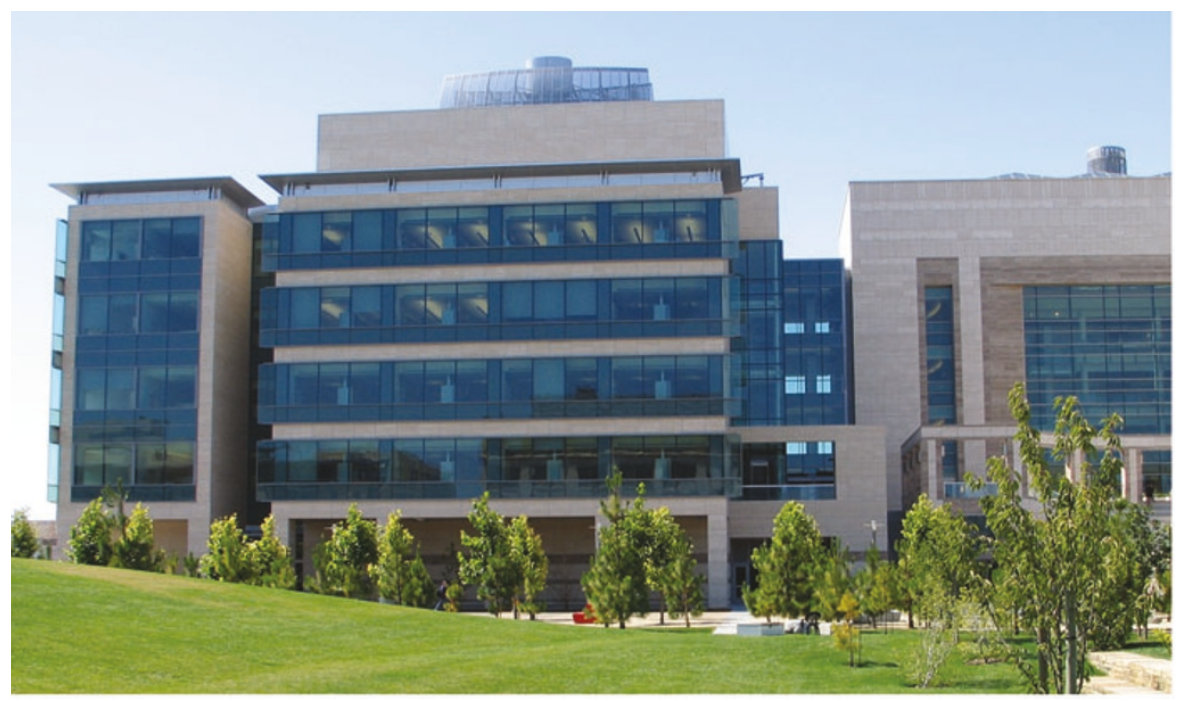

The Life Engineering symposium was held at the California Institute for Quantitative Biomedical Research on the UCSF Mission Bay Campus.

Institute for Quantitative Biomedical Research Institute (QB3), the Lawrence Berkeley National Laboratory (LBNL) Department of Synthetic Biology and the University of Oxford. Here, we highlight the applications and themes that emerged at this meeting.

\section{Live therapeutic agents}

In the campy 1960s science fiction movie Fantastic Voyage, a team of doctors and their submarine are shrunk and injected into a patient's artery. Using a laser, they vaporize a blood clot, after which they are expelled through the patient's tears. Keeping in mind the goal of actually making an automated miniature therapeutic robot, there has been considerable progress in engineering microscopic robots and computers and in harnessing existing biological systems.
Miniature manipulators of the human body already exist. Pathogenic bacteria and viruses are experts at identifying specific cell types and organs, can evade immune responses and can manipulate individual cells. John Pawelek (Yale University) demonstrated that wild-type Salmonella typhimurium and Bordetella pertussis localize to tumors after intravenous injection and can impart a therapeutic effect ${ }^{1}$. To improve efficacy, strains of S. typhimurium have been engineered to require adenosine for growth, convert a prodrug into an active form and ameliorate septic shock. In addition, the Pawlek group demonstrated that the bacteria can be remotely controlled after being administered to a patient by using the inducers tetracycline and mitomycin $\mathrm{C}$ to elicit a response in engineered bacteria thriving in a tumor. 
Programming bacteria to have therapeutic functions will require a toolbox of cellular sensors that respond to microenvironments in the body, genetic circuits to integrate this information and the ability to engineer the interaction between a bacterium and mammalian cell. For example, bacteria localized to a tumor are exposed to high cell densities and anaerobic conditions, which could be used as cues for the release of a therapeutic. Chris Voigt of UCSF demonstrated the linkage of anaerobic and cell density sensors to the induction of invasin, which enables non-pathogenic bacteria to adhere to and invade mammalian cells. Under these conditions, the bacteria attacked a wide variety of cancerous cell lines in culture.

Viruses can also have therapeutic properties. By serendipity, Craig Meyers (Pennsylvania State University) discovered that the harmless human virus AAV2 kills a remarkably broad range of cancer cell lines but does not affect primary cells. AAV has also been used as a vector for gene therapy. Patients with cancers affected by AAV tend not to have existing immunity to $\mathrm{AAV}$, increasing the potential as a therapeutic agent. However, the wild-type virus has a number of drawbacks, including preexisting immunity, delivery efficiency, distribution in the body, production and cell type targeting. Using directed evolution, David Schaffer of the University of California, Berkeley (UCB) found capsid mutants that overcame some of these limitations. One mutant had a 100-fold improvement in avoiding immunity, and this enhanced the delivery of the erythropoietin gene to mice.

Therapeutic viruses could be engineered to interfere with an infection of harmful viruses. Adam Arkin (UCB) outlined a strategy to engineer a lentivirus that interacts with HIV-infected cells to reduce the HIV viral setpoint in the blood and thus prevent the transgression of the disease to AIDS ${ }^{2,3}$. They propose to gut HIV to produce a harmless lentivirus, remove the capsid genes such that it requires a coinfection with HIV in a cell to reproduce, and add components to turn off HIV. They described a theoretical model showing the parameter regime under which the therapeutic virus spreads and reduces the HIV viral setpoint to a level that does not cause AIDS.

\section{Microbial drug factories}

Tools from synthetic biology will have an impact on metabolic engineering, in which the goal is to create an organism that produces a maximum of a desired chemical. One application is to engineer bacteria to synthesize a drug or precursor compound inexpensively. By combining metabolic pathways from bac- teria, yeast and the plant Artemisia annua, Jay Keasling of UCB created a bacterium that synthesizes large quantities of amorphadiene, a precursor of the antimalarial drug artemis$i n i n^{4}$. The production of amorphadiene was maximized by (i) codon optimizing the plant enzyme amorphadiene cyclase using DNA synthesis (142-fold improvement), (ii) incorporation of a heterologous yeast mevalonate pathway (30-fold) and (iii) optimization of the intragenic messenger RNA (mRNA) structures of the synthetic mevalonate operon (sevenfold). These improvements have brought the cost of artemisinin production to a sufficient level to broaden its use in the developing world. A similar engineering approach could be used to optimize other therapeutic terpenes such as taxol, which is used for cancer treatment, and prostratin, which is used in clinical trials for HIV treatment.

\section{Programming stem cells}

Stem cells are able to proliferate and differentiate into many different cell types and are a natural repair mechanism in the body. An understanding of the mechanisms of this process will enable the use of stem cells as a therapy to replace cells destroyed by disease or to grow new tissues. Stem cells can be programmed in several ways. First, their behavior can be tuned by controlling the properties of a well-characterized external microenvironment. Second, synthetic circuits can be introduced that control the activity of central signaling proteins.

David Schaffer presented the construction of a fully characterized external matrix to grow neural stem cells. One of the gel polymers was modified to display a small domain (RGD) from the large protein laminen and was shown to elicit a similar proliferative and differentiation response in the cells. Christopher Chen (University of Pennsylvania) elegantly demonstrated that mesenchymal stem cell commitment to different lineages can be controlled by varying the mechanical environment encountered by the cells. For example, fat cells were generated when the adhesion was limited to a small island, whereas bone cells were formed when the cells were allowed to spread on a larger island ${ }^{5}$. The Chen group also showed that the shapes of multicellular sheets can be used to direct proliferation at specific locations (for example, at corners and valleys) owing to localized tensile stresses generated by the shapes ${ }^{6}$.

A number of signaling proteins can be used as 'switches' in controlling stem cell proliferation and differentiation. David Schaffer showed that the sonic hedgehog protein (Shh) controls whether neural stem cells undergo proliferation or differentiation ${ }^{7}$. Christopher Chen demonstrated that the RhoA GTPase can be engineered to control the differentiation of stem cells into fat (constitutively off) or bone (constitutively on) owing to its role in regulating cell contractility. These outputs can be engineered to respond to heterologous signals. For example, Wendell Lim of UCSF engineered the DH domain of intersectin, which controls filapodia formation, to respond to phosphorylation by protein kinase A (PKA). A synthetic switch was constructed by fusing DH to a PDZ domain and a PDZ substrate that can be phosphorylated by PKA. This fusion protein was then introduced into cells and was induced by the addition of the PKA-activator cAMP, which led to the controllable formation of filapodia.

\section{Biosensors}

RNA has the capability to bind to small molecules and regulate gene expression, making it an ideal substrate for designing biosensors. Christina Smolke (California Institute of Technology) demonstrated that cell-based biosensors can be built by linking a RNA aptamer that binds a small molecule ligand with a piece of RNA that inhibits or activates translation $^{8}$. This was beautifully demonstrated by showing a caffeine sensor that could differentiate between espresso, coffee and decaf. Andy Ellington (University of Texas at Austin) showed a similar design in which an aptamer was fused to a ribozyme such that the catalytic activity was regulated by the binding of a small molecule ${ }^{9}$. Niles Pierce (California Institute of Technology) demonstrated that a biosensor can be constructed by fusing a DNA aptamer to a domain that triggers a hybridization chain reaction, which produces a nucleotide polymer that can be detected by colorimetry, gels or fluorescence ${ }^{10}$. All of these sensors involved simple, modular designs that mechanically link the input with an output response.

Synthetic sensors can also be constructed by fusing an extracellular input domain of a protein to an intracellular signal transducer domain. This strategy was used by the synthetic biology team from the University of Texas at Austin and UCSF, who designed a strain of E. coli that can record a pattern of light that is shown on a bacterial lawn ('bacterial photography'), as described by Edward Marcotte. The light sensor was constructed by fusing the extracellular domain of a cyanobacterial phytochrome with the intercellular domain of an E. coli histidine kinase. A similar approach was described by John Spudich (University of Texas at Houston) in the construction of a phototactic strain of E. colit ${ }^{11}$. Spudich also described a procedure for engineering $E$. coli 
to use light for energy by incorporating the light-driven proton pump proteorhodopsin in their membranes.

\section{Genetic circuits}

Programming cells will require the integration of signals from multiple sensors. Receiving information from multiple sources provides greater specificity when linking gene expression to an environmental niche. An AND gate, in which the output is activated only when all inputs are on, is a particularly useful circuit. Presenters described several new designs for AND gates using DNA, RNA and protein components that functioned in vitro, within bacteria and in eukaryotic cells. Using principles from DNA computing, Ehud Shapiro (Weizmann Institute of Science) demonstrated an AND gate that identifies four 'symptom' inputs (abnormal up- or downregulation of specific mRNA levels) and releases a nucleotide drug in response ${ }^{12}$.

To program bacteria, an AND gate that responds to the activation of two input promoters would be useful in linking the outputs from multiple biosensors and various pathways. Chris Voigt showed such a circuit, in which an input drives an activator modified to contain an amber stop codon, and the second promoter drives the transcription of the transfer RNA (tRNA) amber suppressor. In eukaryotic cells, logic gates can be built by rearranging protein-protein interaction domains. Wendell Lim built a variety of logic blocks using modular domains (such as PDZ and SH3). A relatively small library of designs resulted in a broad range of logic gate behaviors, including AND, OR and antagonistic functions ${ }^{13}$. This work was extended to create a three-input AND gate. In addition, it was shown that the transfer function of a protein switch could be tuned from a graded to a switch-like response by increasing the number of domains associated with one input. Christina Smolke combined a theophylline-binding RNA sensor with an RNA hairpin structure that acts as a temperature sensor to produce a circuit in yeast that responds to a small-molecule ligand only above certain temperatures.

Obtaining programmable multicellular organization will require the use of genetic circuits that control cell-cell communication. Lingchong You (Duke University) described a genetic circuit that regulates bacterial population density using a quorum sensor linked to the expression of a toxic protein ${ }^{14}$. Moving up a level in complexity, he demonstrated the use of two quorum sensors to create a predatorprey system. These circuits could be used to program temporal-spatial patterns into cells, which has applications in designing biofilms and growing synthetic tissue.

\section{Rational biomolecular design}

There have been tremendous advances in the ability to design the sequence of a biological polymer to fold into a target structure. Tanja Kortemme (UCSF) presented a computational algorithm that generates pairs of proteins that bind to each other ${ }^{15}$. This is done in silico by destabilizing the interface and then reengineering the partner to optimize the binding affinity. This algorithm was successfully applied to the redesign of a PDZ binding interaction, which could be used to create circuits and rewire signaling pathways.

In creating synthetic cells, it will be important to understand how the composition of lipids in the membranes affects the physics of micelle formation. This was explored by Mark Sansom (Oxford University), who used molecular dynamics simulations to study the self-assembly of lipids and membrane proteins into micelles and bilayers ${ }^{16}$.

Niles Pierce described algorithms that can be used to design nucleotide sequences to program synthetic mechanical functions into RNA and DNA. As an example, he showed the design of a system of DNA fragments that are able to self-assemble into a motor analogous to kinesin and take the first steps along a piece of DNA ${ }^{17}$. This work opens up the possibility that mechanical functions can be programmed into cells using nucleotide sequences.

\section{Directed evolution as a tool}

Intelligent designers sometimes resort to using in vitro evolution as a rapid method to engineer a biological system. At the symposium, directed evolution emerged as a common method that was used successfully to improve metabolic pathways, mRNA switches, viruses and genetic circuits. Screening libraries of genetic circuits requires a different strategy from evolving a single protein or enzyme. Circuits are characterized by their transfer function, which captures their output over a range of input conditions. At a minimum, this requires screening in two conditions: in the presence and absence of input signal.

Several new positive-negative selection strategies were proposed that are able both to search through large libraries and to identify functional circuits. Yohei Yokobayashi (University of California, Davis) demonstrated a dual selection where the $\mathrm{ON}$ and OFF states of a switch activated the expression of different antibiotic resistance genes. This strategy was applied to the evolution of a genetic inverter. Chris Voigt presented a positive-negative selection strategy that used the Yersinia pseudotuberculosis protein invasin as an output and mammalian cells as sequestering devices.
Selection strategies also have their pitfalls because cells are very good at finding unintended pathways to survive. This principle emerged in a project described by Andy Ellington in which a selection scheme that intended to create a ribozyme-based genetic circuit ended up creating novel secondary structures that deactivated the circuit, which had unintended toxicity. It was found that the hairpins deactivated ribosomes-a principle that he is now using to regulate the number of ribosomes per cell as a mechanism to control cellular noise.

\section{Ethics, security and society}

At the symposium, four talks directly addressed ethical, safety and security issues. Laurie Zoloth (Northwestern University), a bioethicist, spoke about the ethical concerns raised by the work, how they arise from larger social responses to science and technology, how such concerns give raise to public discourse and how that discourse can lead to varying models of regulation. Paul Rabinow (UCB), an anthropologist, discussed safety and security concerns in light of the history of prior regulation of science. Two scientists, Andy Ellington (University of Texas, Austin) and Roger Brent (Molecular Sciences Institute), advocated the importance of paying attention to student training and to articulating possible future applications that promise great benefit to society. The following is a summary of the themes from these presentations and the discussions afterward.

All new technologies can be used to cause harm. Moreover, errors, mistakes, and, indeed, dual use (meaning the use for both social welfare and for weaponry) of all technology, including synthetic biology, is inevitable. However, the potential for both malevolent and beneficent use cannot unilaterally preclude basic research. Using this as an argument to stop research progress would have halted all technology since the development of metallurgy. The problem of dual use, or of unintended consequences, is not intrinsic to synthetic biology, but rather due to a widening of the number of people that can work with DNA. Given that technology such as recombinant DNA has been widely used for 25 years, the questions are, how can we minimize the risks? How do we best prepare for contingencies? Have new technologies emerged that require revisiting previous strategies?

As applied to recombinant DNA, these concerns were addressed by the 1975 Asilomar meeting. Asilomar succeeded for two reasons. First, it reduced the danger to a question of safety, which converted the issue from a political one to a technical and hence manageable one. Scientists at the meeting then devised 
a scheme involving graduated levels of containment and laid out a series of experiments that could give answers that would support the relaxation of the level of containment. However, given the current focus on security, the attempt to reduce questions of risk to questions of safety is not sufficient. Second, they articulated the societal benefits that can come from recombinant DNA. They identified three: gene therapy, crops that will fix their own nitrogen and production of recombinant protein drugs, including insulin and interferon. Even though only one of these applications has so far been successful, its real benefits have helped to allay fear. Following the example of Asilomar, there needs to be a coherent (and articulate) scientific community in synthetic biology that works to mitigate the risks while convincingly communicating realistically possible benefits.

Training programs will train a cadre of young synthetic biologists that have the tools necessary to handle emerging threats, humility in the face of the risks, an understanding of the problem and a commitment to education, prudence and self-regulation. This bottom-up structure is ultimately better than trying to limit the technology itself, leaving us with a small group of trained researchers and no regulatory structure. Several educational programs are being built to address this challenge. Drew Endy (Massachusetts Institute of Technology) presented an undergraduate class that brings hands-on experience in designing, building and testing cells that can perform synthetic tasks. There is also an annual nationwide genetic engineering competition (iGEM) in which teams of students compete to engineer a synthetic organism. This year, fourteen schools are participating. As an educational tool, these programs have focused on toy problems in cellular control. To create a base to deal with emerging problems, we will need to expand the curriculum to include applications such as vaccine development, energy production and materials.

All research will also need a careful public system for oversight. It is important to understand the reasonable public understanding of the risk inherent in this research. Laurie Zoloth presented a list of the reasons behind the public's concern about synthetic biology. The arguments for caution emerge from many political, social and theoretical stances across the American spectrum. Political skepticism arises from the political left, which raises concerns about any genetic alteration, and the political right, which raises questions about the scientific project itself. The argument can be summarized as follows: first, there is a view that nature is a morally good, hierarchical and stable system that is instantiated in large part in DNA. Natural order can be violated when, for example, the species barrier is broken down in the creation of chimeric or recombinant DNA. Similarly, building synthetic biological systems suggests a mastery over finitude, and some raise the fear that such a mastery is an attempt to avoid core aspects of the human condition — suffering and death—which, they argue, is a definition of our species. Many fear a 'slippery slope' in which such technology could redefine humanity in disturbing, unjust or mechanistic ways. Others note that dual use and mistakes are inevitable and warn against catastrophic secondary effects with enormous consequences. Lastly, others warn about the conflict of interests inherent in science that is linked to patent, profits and markets, fearing that the marketplace will distort the science.

Roger Brent made the point that convincing the public of the benefits of synthetic biology will require the articulation of a core set of applications, similar to those that arose out of Asilomar, on which the nascent community might actually deliver. It would be helpful in this regard to diminish the hype that has entered into these discussions, some of which has arisen in order to attract funding. False promises will erode public confidence and the credibility of researchers among other scientists. Keeping this in mind, a series of challenges were proposed.

Bulk chemical synthesis. The future chemical industry will use glucose as a feedstock, not oil. Accelerating the decrease in the price of glucose will hasten the day when it becomes less expensive than hydrocarbons, and it would be useful for synthetic biologists to hasten the intersection of those two trend lines: the ' green chemical industry' takeoff point.

Energy. Cells and organisms can be used to produce fuels, and electron-handling machinery from photosynthetic reaction centers might find use in fuel cells built from biologi- cal components. In addition, of course, cells have natural photosynthetic processes that can be harnessed in these applications.

Fine chemical synthesis and therapeutics. Keasling's work illustrates the possible utility of developing cells that can make precursors to one sort of chemical backbone and potential great utility of such cells in synthesizing drugs that are directed against malaria, a disease of the developing world. Others may well extend it to other backbones and thus to other diseases. It may also be possible to use biological components to create smart drug delivery devices.

Research. A potential 5- to 10-year project might be to make logic and output (DNA writing) circuitry to develop a combination cell cycle counter and lineage trace that can be used to identify the developmental lineage of every cell in the body of a mammalian organism.

These inspirational goals could serve to shape the field and to define credible, social benefits for the work. Even modest success in one of these applications would have a tremendous impact on society.

1. Pawelek, J., Low, K.B. \& Bermudes, D. Lancet Oncol. 4, 548-556, 2003 (2003).

2. Weinberger, L., Schaffer, D.V. \& Arkin, A.P. J. Virol. 77 10028-10036 (2003).

3. Weinberger, L.S., Burnett, J.C., Toettcher, J.E., Arkin, A.P. \& Schaffer, D.V. Cell 122, 169-182 (2005)

4. Martin, V.J.J., Pitera, D.J., Withers, S.T., Newman, J.D. \& Keasling, J.D. Nat. Biotechnol. 21, 796-802 (2003).

5. McBeath, R., Pirone, D., Nelson, C.M., Bhadriraju, K. \& Chen, C.S. Dev. Cel/ 6, 483-495 (2004).

6. Nelson, C.M. et al. Proc. Natl. Acad. Sci. USA 102 11594-11599 (2005).

7. Lai, K., Kaspar, B.K., Gage, F.H. \& Schaffer, D.V. Nat. Neurosci. 6, 21-27 (2003).

8. Bayer, T.S. \& Smolke, C.D. Nat. Biotechnol. 23, 337343 (2005)

9. Levy, M. \& Ellington, A.D. Chem. Biol. 9, 417-426 (2002).

10. Dirks, R.M. \& Pierce, N.A. Proc. Natl. Acad. Sci. USA 101, 15275-15278 (2004).

11. Jung, K.H., Spudich, E.N., Trivedi, V.D. \& Spudich, J.L. J. Bacteriol. 183, 6365-6371 (2001).

12. Benenson, Y., Gil, B., Ben-Dor, U., Adar, R. \& Shapiro, E. Nature 429, 423-429 (2004).

13. Dueber, J.E., Yeh, B.J., Chak, K. \& Lim, W.A. Science 301, 1904-1908 (2003).

14. You, L., Cox, R.S., Weiss, R. \& Arnold, F.H. Nature 428 868-871 (2004).

15. Kortemme, T. et al. Nat. Struct. Mol. Biol. 11, 371-379 (2004).

16. Bond, P.J., Cuthbertson, J.M., Deol, S.S., \& Sansom, M.S. J. Am. Chem. Soc. 126, 15948-15949 (2004).

17. Shin, J.S. \& Pierce, N.A. J. Am. Chem. Soc. 126 10834-10835 (2004) 